\title{
CONVERGENCE, SUMMABILITY, AND UNIQUENESS OF MULTIPLE TRIGONOMETRIC SERIES
}

\author{
BY J. MARSHALL ASH ${ }^{1}$ AND GRANT V. WELLAND ${ }^{2}$ \\ Communicated by Paul J. Cohen, August 25, 1970
}

1. Relationships between methods of convergences and the growth of coefficients. It was shown by Paul J. Cohen [1] that if a multiple trigonometric series converges regularly at almost every point of the $k$-torus $T^{k}=[-\pi, \pi] \times \cdots \times[-\pi, \pi]$, then its coefficients $a_{n}$ $=a_{n_{1}}, \cdots, n_{k}$ cannot exhibit exponential growth. A particular form of regular convergence is square convergence. Consideration of double series of the form

$$
\sum_{n=1}^{\infty} \phi(n)(1-\cos x)^{n} e^{i n y}
$$

shows that Cohen's seemingly gross estimates cannot be improved. For by a suitable choice of the $\phi(n)$ the series may be made square convergent almost everywhere while having coefficients which grow faster than any given sequence whose growth is less than exponential.

THEOREM 1. If a multiple trigonometric series converges unrestrictedly rectangularly on a set, then the coefficients are necessarily bounded; furthermore, $a_{n}=a_{n_{1}}, \cdots, n_{k} \rightarrow 0$ as $\min \left\{\left|n_{1}\right|, \cdots,\left|n_{k}\right|\right\}=\|n\| \rightarrow \infty$.

Again this theorem is best possible. The proof is by induction and makes use of

Lemma 1. If a polynomial $P\left(e^{i x}\right)$ of degree $n$ is bounded for all $x \in E \subset[0,2 \pi)$ by a bound $B$, where $|E|=$ Lebesgue measure of $E=\delta>0$, then there is a number $c=c(\delta, n)$ such that $\left|P\left(e^{i x}\right)\right| \leqq c$ for every $x$.

The lemma is an easy consequence of the Lagrange interpolation formula and a lemma of Paul Cohen's [1, p. 41]. Another consequence of Lemma 1 is

AMS 1970 subject classifications. Primary 42A92, 42A48, 42A20, 42A24, 40B05: Secondary 40G10, 40A05, 40D15.

Key words and phrases. Square summable, unrestrictedly rectangularly convergent, spherically Abel summable, $C(0, \beta)$ summable, uniqueness, coefficient growth, Riemann summable.

1 Research partially supported by NSF Grant GP 14986.

2 Research partially supported by NSF Grant GP 9123.

Copyright @ 1971, American Mathematical Society 
THEOREM 2. If a multiple trigonometric series $\boldsymbol{\Sigma}$ converges unrestrictedly rectangularly on a subset $E$ of $T^{k}$, then there is a set $F \subset E$, $|F|=|E|$ such that all the reclangular partial sums of $\Sigma$ are bounded on $F$. (The bound may vary from point to point.) In particular, if $E=T^{k}$, then $F=E$.

To appreciate that this theorem is trivial only if $k=1$, the reader should consider the numerical double series given by $a_{0 n}=n, a_{1 n}=-n$, $n=1,2,3, \cdots, a_{m n}=0$ otherwise which converges unrestictedly rectangularly to 0 , but has unbounded rectangular partial sums. The proof of Theorem 2 is also an induction which depends heavily on Lemma 1.

2. Relationships between modes of convergence and summability.

THEOREM 3. If a multiple trigonometric series $\Sigma$ converges unrestrictedly rectangularly on a set $E$, then $\Sigma$ is spherically Abel summable to the same values on $F$, where $E$ and $F$ are as in Theorem 2.

This theorem is an easy consequence of Theorem 2 and the fact that Pringsheim convergence (unrestricted rectangular convergence together with bounded rectangular partial sums) implies spherical Abel summability for numerical multiple series. (We say $\sum a_{n}$ is spherically Abel summable to $s$ if $\sum a_{n} e^{-\left.\right|^{n} \mid h}=A(h)$ exists for every $h>0$ and $\lim _{h \rightarrow 0+} A(h)=s$.)

Theorem 3 is somewhat surprising since different modes of convergence are often incompatible. For example, the double series

$$
\sum_{p=2}^{\infty} 3 p \exp \left[i p^{2} x\right](\sin y)^{2 p} \exp \left[i\left(p^{2}-2 p\right) y\right]
$$

is square convergent almost everywhere but restrictedly rectangularly convergent at no point; while the double series

$$
\sum_{p=1}^{\infty} 2^{p} \exp \left[i 4^{p} x\right](\sin y)^{2^{p}}
$$

is restrictedly rectangularly convergent almost everywhere but both circularly and triangularly divergent everywhere. We do have, however,

THEOREM 4. If a multiple trigonometric series converges on $E$, then it is $(C, 1,1)$ summable on $F$, where $E$ and $F$ are as in Theorem 2.

The proof is again Theorem 2 together with a classical theorem concerning numerical series. There are many similar classical the- 
orems in which the hypothesis of bounded partial sums may be dropped. It would be repetitious to list any more of them here.

\section{Uniqueness of multiple trigonometric series.}

THEOREM 5. Let the double trigonometric series $\Sigma$ be unrestrictedly rectangularly convergent everywhere on $T^{2}$ to the finite-valued Lebesgue integrable function $f(x, y)$. Then $\Sigma$ is the Fourier series of $f$.

The proof of Theorem 5 involves the use of a uniqueness theorem concerning spherical Abel summability due to Victor Shapiro ([5] or $[6, \mathrm{pp} .65-78])$. The hypotheses in Shapiro's theorem that must be verified are

(i) $\Sigma$ is spherically Abel summable everywhere to $f(x, y)$, and

(ii) the sum of the moduli of the coefficients of $\Sigma$ whose indices lie in an annulus of thickness one and radius $R$ is $o(R)$ as $R$ tends to infinity.

The first hypothesis is satisfied because of Theorem 3 while the second follows from Theorem 1. Condition (ii) does not follow from Theorem 1 if the dimension $k$ is $>2$ which is why the theorem is stated only in two dimensions. A somewhat more general version of Theorem 5 involving upper and lower limits actually holds but it is too complicated to state here. In higher dimensions, we have the following uniqueness theorem.

Theorem 6. Suppose for every $\left(x_{1}, \cdots, x_{k}\right)=\mathrm{x}$,

$$
\begin{array}{r}
\lim _{\left\|\left(N_{1}, \cdots, N_{k}\right)\right\| \rightarrow \infty} \sum_{n_{1}=0}^{N_{1}} \cdots \sum_{n_{k-1}=0}^{N_{k}-1} \sum_{n_{k}=-N_{k}}^{N_{k}} a_{n_{1}}, \cdots, n_{k} \\
\exp \left[i\left(n_{1} x_{1}+\cdots+n_{k} x_{k}\right)\right] \\
=\sum a_{n} \exp [i n \cdot \mathrm{x}]=0 .
\end{array}
$$

Then all the $a_{n_{1}}, \ldots, n_{k}$ are zero.

The statement is given in a fairly specific fashion since it is not clear at the present time whether the restriction that all but one of the sums be one-sided is necessary. A uniqueness theorem for multiple trigonometric series of power series type is an immediate corollary.

To prove Theorem 6 form a sequence of functions $L_{0}(x)=$ $\sum a_{n} \exp \left[i_{n} \cdot \mathrm{x}\right], L_{1}(\mathrm{x}), \cdots, L_{k}(\mathrm{x})$ where each $L_{i}$ is obtained by two formal integrations in the $i$ th coordinate. The facts that the second $i$ th partial symmetric derivative of $L_{i}$ is equal to $L_{i-1}$ and that $L_{i}$ is a continuous function of its $i$ th variable are used to establish that the continuous function $L_{k}(x)$ has a special form from whence it follows that $L_{k}$ and hence $L_{0}$ have all coefficients equal to zero. 
Another approach to the theory of uniqueness may be made by considering $L_{k}(x)$ directly and working with a $k$ th symmetric derivative directly. This method, which has been pursued by Geiringer [2] and Žak [8] does yield some interesting partial results but not as yet a theory of uniqueness.

A fourth approach to uniqueness is via generalized "integrals" wherein the first "integral" is a vector (Shapiro [7]), the second "integral" is a matrix, the third a three tensor, and so forth. A partial result arising from this study is that

$$
\lim _{n \rightarrow 0} \sum_{m=0}^{\infty} \sum_{n=0}^{\infty} a_{m n}\left(\frac{m \sin m h+n \sin n h}{\left(m^{2}+n^{2}\right) h}\right)^{\alpha}=s
$$

whenever $\alpha>2$ and $\sum \sum a_{m n}=s$ in the sense of Pringsheim.

4. On the almost everywhere summability of double Fourier series. The Fourier series $S[f]$ of $f(x, y) \in L\left(T^{2}\right)$ is said to be summable $(C, \alpha, \beta)$ at $(x, y)$ if

$$
\lim _{\|(m, n)\| \rightarrow \infty} \frac{1}{\pi^{2}} \int_{-\pi}^{\pi} \int_{-\pi}^{\pi} f(x-s, y-t) K_{m}^{\alpha}(s) K_{n}^{\beta}(t) d s d t=f(x, y),
$$

where $K_{m}^{\alpha}(s)$ is the $\alpha$ th Fejér kernel. (See Zygmund [9, p. 94].)

Jessen, Marcinkiewicz, and Zygmund [4] have proved that if $\alpha>0$ and $\beta>0$, and if $f \in L \log { }^{+} L$ on $T^{2}$, then $f$ is summable $(C, \alpha, \beta)$ at almost every point of $T^{2}$. If $\beta=0$, we set

$$
K_{n}^{0}(t)=D_{n}(t)=\frac{\sin \left(n+\frac{1}{2}\right) t}{2 \sin t / 2} .
$$

THEOREM 7. If $f \in L(\log +L)^{2}$ on $T^{2}$ and if $\alpha>0$, then $f$ is summable $(C, \alpha, 0)$ almost everywhere on $T^{2}$.

The proof uses maximal functions and is an easy corollary of a onedimensional maximal theorem of Hunt [3] concerning convergence in the theory of functions of one variable.

\section{BIBLIOGRAPHY}

1. Paul J. Cohen, Topics in the theory of uniqueness of trigonometrical series, Thesis, University of Chicago, Chicago, Ill, 1958.

2. H. Geiringer, Trigonometrische Doppelreihen, Monatsh. Math. Phys. 29 (1918), 65-144.

3. R. A. Hunt, On the convergence of Fourier series, Proc. Conf. Orthogonal Expansions and their Continuous Analogues (Edwardsville, Ill., 1967), Southern Illinois Univ. Press, Carbondale, Ill., 1968, pp. 235-255. MR 38 \#6296. 
4. B. Jessen, J. Marcinkiewicz and A. Zygmund, Note on the differentiability of multiple integrals, Fund. Math. 25 (1935), 217-234.

5. V. L. Shapiro, Uniqueness of multiple trigonometric series, Ann. of Math. (2) 66 (1957), 467-480. MR 19, 854; 1432.

6. - Fourier series in several variables, Bull. Amer. Math. Soc. 70 (1964), 48-93. MR 28 \#1448.

7. - The approximate divergence operator, Proc. Amer. Math. Soc. 20 (1969), 55-60. MR 38 \#4918.

8. I. E. Žak, On Riemann summability of double numerical series, Soobšc. Akad. Nauk Gruzin. SSR 13 (1952), 587-593. MR 14, 1079.

9. A. Zygmund, Trigonometric series. Vol. I, 2nd rev. ed., Cambridge Univ. Press, New York, 1959. MR 21 \#6498.

DePaul University, Chicago, Illinois 60614 